\title{
Einstein-Bohr Controversy After 75 Years, Its Actual Solution and Consequences
}

\author{
Miloš V. Lokajíček \\ Institute of Physics of the AS CR, Prague \\ Czech Republic
}

\section{Introduction}

The contemporary fundamental physics has been based fully on phenomenological models starting from the Copenhagen quantum mechanics proposed by Bohr in 1927 (1). However, the Copenhagen model was criticized strongly by Einstein and collaborators (2) from the point of view of ontological interpretation of matter world, i.e., from the grounds on which the whole physical concept was based by Galileo and Newton. Einstein called the attention (with the help of a Gedankenexperiment) to the fact that immediate interaction between different matter objects (in principle between different measuring devices) at great mutual distances was required in Bohr's theory (the effect being unacceptable from the ontological point of view and denoted now usually as entanglement). However, the criticism of Einstein was refused strongly by Bohr (3). And the physical community has accepted fully Bohr's arguments (that the rules in microworld differ from those of macroworld) even if Einstein did not changed his standpoint till the end of his life, having been aware of the correctness of his conclusion (see also (4)).

Later (on the basis of Bohm's paper (5)) two different quantum alternatives were considered, i.e., the Copenhagen alternative and the so called hidden-variable theory. Some differences between these two alternatives started to be discussed when Bell (6) derived his famous inequalities. These inequalities should have been valid for some combinations of four probabilities of the passage of two particles with opposite spins through two polarizers; the original EPR (Einstein-Podolsky-Rosen) Gedankenexperiment having been slightly modified. To obtain the given inequalities Bell had to assume for individual probabilities to be rather strongly mutually correlated; and it has been believed that the given inequalities have been valid in the framework of the hidden-variable theory. It was, therefore, expected that their violation (or not) in an experiment might have helped in deciding between the two given quantum alternatives.

As to the original Gedankenexperiment of Einstein it was based on the coincidence detection measurement of two decay objects emitted in a decay of an unstable particle and going in opposite directions. The basic proposal of this experiment was then modified to be possible actually to perform it. And the coincidence transmission probabilities of two photons having opposite spins and running in opposite directions through two polarizers were being established:

$$
\left\|<--\left.\right|^{\beta}---o---\left.\right|^{\alpha}-->\right\|
$$


where the axes of individual polarizers were deviated by angles $\alpha$ and $\beta$ from some common zero position. The corresponding measurements were performed in the seventieth years of the past century and ended practically in 1982 (see (7)) with the following results:

- the inequalities of Bell's combinations of coincidence probabilities have been violated;

- the measured values of individual coincidence probabilities have fulfilled approximately the Malus law (see Sec. 4 of this chapter);

and both the results have been interpreted as decisive support for the Copenhagen quantum mechanics.

However, the given conclusion has been based on several mistakes. Both the quantum alternatives (starting from the same Schrödinger equation) have been regarded as two different interpretations of one common theoretical model. In fact each of these interpretations has been based on discrepant sets of assumptions, representing two fully diverse theories (see Sec. 2).

It has been then possible to show further that in the interpretation of the experimental results gained on the basis of the EPR experiments mainly two mistakes have played important roles (8) (see also $(9 ; 10)$ ):

(i) It has been believed all the time that Bell's inequalities have held in the framework of hidden-variable theory. However, they were based on the assumption holding only in the framework of classical physics, which means that no quantum alternative may be excluded on the basis of the results of EPR experiments (see Sec. 3).

(ii) As to the measured Malus law it was argued by Belinfante (11) that a very different result should be obtained in the performed coincidence EPR experiments. However, also this argument has been based on mistaking assumption (see Sec. 4).

It follows, therefore, that contrary to common conviction any of two quantum alternatives cannot be excluded on the basis of the results of EPR experiments. Even if the hidden-variable theory should be at least partially preferred (the lower limit not having been overpassed, see Sec. 3), one must look for other ways how to decide between them.

And it has been possible to demonstrate that the hidden-variable theory being practically equivalent to the Schrödinger equation (Bohr's deforming assumptions not having been added) should be preferred not only to the Copenhagen alternative but also to the classical physics:

(i) The Copenhagen quantum mechanics is to be practically excluded as it involves some important internal contradictions (as it will be shown in Sec. 5) that have been interpreted until now as quantum paradoxes.

(ii) There are also some experimental data that are in clear contradiction to the predictions of Copenhagen quantum mechanics; see Sec. 6.

(iii) And finally, the basic solutions of Schrödinger equation (determined always with the help of one Hamiltonian eigenfunction only) give the same results as classical physics, which prefers this equation (or hidden-variable theory) to be applied to the whole matter reality (microscopic as well as macroscopic); see Sec. 7.

The physical interpretation of Schrödinger equation was strongly influenced by the requirements put by Bohr on the shape of corresponding Hilbert space. If the physical content is to remain undeformed the Hilbert space must consist at least of two individual subspaces being bound by evolution operator as it was shown already by Lax and Phillips $(12 ; 13)$ in 1967 and as it will be discussed in Sec. 8 . 
The evolution of physics in the last centuries was strongly influenced by the philosophical ideas that started to influence any thinking of human society from the beginning of the modern age and differed fundamentally from the earlier ontological thinking. The corresponding story will be mentioned in Sec. 9.

Main conclusions and consequences will be then summarized in the last section (Sec. 10).

\section{Two quantum alternatives and corresponding assumptions}

It is possible to say that the Copenhagen quantum mechanics has been based in principle on four following assumptions:

- first, it is the validity of time-dependent Schrödinger equation (14)

$$
i \hbar \frac{\partial}{\partial t} \psi(x, t)=H \psi(x, t), \quad H=-\frac{\hbar^{2}}{2 m} \triangle+V(x)
$$

where Hamiltonian $H$ represents the total (kinetic and potential) energy of a given physical system and $x$ represents the coordinates of all matter objects;

- physical quantities are expressed as the mean values of corresponding operators at time $t$ :

$$
A(t)=\int \psi^{*}(x, t) A_{o p} \psi(x, t) d x
$$

where functions $\psi(x, t)$ represent vectors and $A_{o p}$ operators in some Hilbert space, the structure of which having been left unspecified (in the framework of this assumption);

- in the Copenhagen alternative the corresponding Hilbert space has been then required to be spanned on one set of Hamiltonian eigenfunctions $\psi_{E}(x)$ :

$$
H \psi_{E}(x)=E \psi_{E}(x)
$$

- any vector of such a Hilbert space has represented a so called "pure" state of a corresponding physical system, which has meant that the mathematical superposition principle (holding in any Hilbert space) has been interpreted in physical sense.

While the Hilbert space has remained unspecified in the second assumption, the first two assumptions have been shared also by the hidden-variable theory. However, to get the whole assumption set belonging to the hidden-variable theory the third and fourth earlier assumptions must be refused and substituted by:

- the Hilbert space must be adapted to a given physical problem, consisting of some mutually orthogonal subspaces and being at least doubled in comparison to the third assumption in the preceding assumption set, if the original physical interpretation of Schrödinger time-dependent solution is to be conserved;

- only the vectors of orthogonal basis of extended Hilbert space (represented in principle by eigenfunctions of the Hamiltonian) may be interpreted as "pure" states; any superposition represents always a "mixed" state.

The given quantum alternatives represent, therefore, two quite different theories. They cannot be interpreted in any case as two mere different interpretations of one common mathematical model. Only the Schrödinger equation is shared commonly, its solutions being interpreted 
in quite different ways. In the Copenhagen quantum mechanics the time evolution of any physical system is described as time reversible. In the hidden-variable theory the evolution is irreversible; the former third assumption being refused and the Hilbert space being suitably chosen (i.e., extended) in full agreement with the time-dependent solutions of Schrödinger equation (see Sec. 8).

\section{Assumption in Bell's inequalities}

It was believed since 1964, even if any more detailed analysis was not performed, that the assumption needed for the derivation of Bell inequalities corresponded to the hidden-variable theory. However, it has been shown later (see, e.g., (9)) that the given assumption has corresponded to the classical physics and it has not been possible to attribute it to any quantum alternative.

We shall describe now shortly the essence of the corresponding mistake. As already mentioned the given experiment has consisted in the measurement of coincidence transmission probabilities of two photons having opposite spins and running in opposite directions through two polarizers:

$$
\left\|<--\left.\right|^{\beta}---o---\left.\right|^{\alpha}-->\right\|
$$

Introducing some rather strong correlation conditions between individual transmission probabilities $a_{j}$ and $b_{k}$ Bell (6) (see also (15)) has derived the following inequalities

$$
B=a_{1} b_{1}+a_{2} b_{1}+a_{1} b_{2}-a_{2} b_{2} \leq 2
$$

holding for any two pairs of orientations of both the polarizers (4 different combinations). The inequalities (4) have been then attributed to the hidden-variable alternative (without any deeper analysis). However, the given assumption has been much stronger and has eliminated practically the possibility for photon spins to influence the values of individual transmission probabilities, which corresponds to the original proposal of the given Gedankenexperiment by Einstein.

Any individual probability in the given experiment should be determined in principle by three different parameters: position of the photon source, photon spin direction and polarizer orientation. And the influence of two last parameters has been excluded when the assumption (leading to Bell's inequalities) has been applied to. The given inequalities were derived, of course, in other ways, too; see, e.g., Ref. (15), where instead of one assumption of Bell several weaker assumptions have been made use of. And when some assumptions have corresponded to the hidden-variable alternative one of these assumptions has held always in the classical physics only (see, e.g., (16)).

The given situation may be represented more clearly when the individual probabilities $a_{j}$ and $b_{k}$ are substituted by operators representing individual measurement acts and acting in two different subspaces (corresponding to individual polarizers) of the whole Hilbert space

$$
\mathcal{H}=\mathcal{H}_{a} \otimes \mathcal{H}_{b}
$$

It holds for the expectation values of these operators (see (17))

$$
0 \leq\left|\left\langle a_{j}\right\rangle\right|,\left|\left\langle b_{k}\right\rangle\right| \leq 1 .
$$


Eq. (4) represents then the definition of Bell's operator $B$. And it may be immediately seen that it holds $\left\langle B^{*} B\right\rangle \leq 16$ and/or $\langle B\rangle \leq 4$, which represents the highest possible limit. However, Bell's operator $B$ may exhibit different limits according to basic assumptions concerning the relations holding between individual operators.

According to chosen commutation relations between $a_{j}$ and $b_{j}$ three different limits may be obtained (see (9)):

$$
|B| \leq 2,2 \sqrt{2} \text { or } 2 \sqrt{3} \text {. }
$$

The first limit corresponds to the classical case, when all operators $a_{j}$ and $b_{k}$ commute mutually, i.e., if

$$
\left[a_{j}, b_{k}\right]=0, \quad\left[a_{1}, a_{2}\right]=\left[b_{1}, b_{2}\right]=0 .
$$

The second limit corresponds to the hidden-variable theory, when only the operators belonging to different subspaces commute (no interaction at distance or no entanglement), i.e., if

$$
\left[a_{j}, b_{k}\right]=0 \text { and }\left[a_{1}, a_{2}\right] \neq 0,\left[b_{1}, b_{2}\right] \neq 0 .
$$

And finally, the third limit (and actually the highest one (18)) corresponds to the case when the interaction at distance exists and the operators from different Hilbert subspaces do not commute mutually, i.e., if

$$
\left[a_{j}, b_{k}\right] \neq 0, \quad\left[a_{1}, a_{2}\right] \neq 0, \quad\left[b_{1}, b_{2}\right] \neq 0 .
$$

Only the classical alternative has been, therefore, excluded by the results of EPR experiment. As to the hidden-variable theory it does not contradict the results of EPR experiments (obtained, e.g., by Aspect et al. (7)); the value $2 \sqrt{2}$ not having been overpassed. It is, of course, also the Copenhagen quantum mechanics that has not been excluded at least in principle. And further arguments should be made use of to decide with certainty which of the quantum alternatives represents really the description of microscopic physical reality; see Secs. 5 and 6.

\section{Malus law and EPR coincidence experiment}

One of the arguments supporting the Copenhagen quantum mechanics was added by Belinfante (11) as already mentioned. He tried to express the coincidence probability in the EPR polarization experiment as a function of $(\alpha-\beta)$. It would be possible easily if the individual dependencies $a(\alpha)$ and $b(\beta)$ for single transmission probabilities were known; $\alpha$ and $\beta$ being the angles between photon spin (or photon polarization) and polarizer axis.

However, in fact only the transmission of unpolarized light through two polarizers have been measured

$$
o---\left.\right|^{\alpha}---\left.\right|^{\beta}-->||
$$

and the so called generalized Malus law has been approximately obtained

$$
M(\alpha-\beta)=(1-\varepsilon) \cos ^{2}(\alpha-\beta)+\varepsilon
$$

where $\varepsilon$ represents very small correction. And Belinfante came to the given statement for the coincidence measurement when he put quite arbitrarily $a(\alpha)=\cos ^{2} \alpha$ and $b(\beta)=\cos ^{2} \beta$, which cannot correspond to real conditions; i.e., the Malus formula holding approximately for a polarizer pair was applied to each polarizer. And there is not any reason to expect for 
coincidence measurements the values of the angle dependencies differing significantly from the Malus law, as required by Belinfante.

The given mistake has not represented, of course, the main argument for the then conclusion. This argument followed primarily from the violation of Bell's inequalities. However, Belinfante contributed probably rather strongly to it. In fact the approximate Malus law can be easily obtained in the given EPR experiment if the probabilistic functions $a(\alpha)$ and $b(\beta)$ are suitably chosen in agreement with reality (19). And the second experimental result obtained in 1982 (see Sec. 1) could not represent in principle any contribution to the solution of the given controversy.

\section{Copenhagen alternative and internal discrepancies}

The Copenhagen quantum mechanics has ascribed to the physical reality some properties that are denoted usually as paradoxes (wave-particle duality, tunnel phenomenon, non-local interaction or entanglement, a.s.o.). However, these paradoxes followed practically from some contradictions contained in the corresponding mathematical model. And it is possible to introduce at least three contradictions which may be denoted as decisive (see also (9)):

- Already the former fourth assumption itself (see Sec. 2) concerning the superposition principle has represented very important discrepancy, which has concerned the existence of discrete states in Schrödinger equation. It is evident that if the given assumption has been added all mathematical superpositions in the corresponding Hilbert space should have represented physical states fully equivalent to basic ones (i.e., to pure states) and no quantized (discrete) states might exist in principle in experimental reality. The given problem has been removed in the hidden-variable theory when only basis vectors corresponding to Hamiltonian eigenstates have represented "pure" states and any superposition of theirs has represented a statistical "mixture" (see also Sec. 7).

- The former third assumption has played then important role in the other discrepancy. Already in 1933 Pauli (20) showed that in such a case it was necessary for the corresponding Hamiltonian to have continuous energy spectrum from $-\infty$ to $+\infty$, which disagreed with the fact that the energy was defined as positive quantity, or at least limited always from below; the existence of discrete states having been excluded, too.

- In 1964 Susskind and Glogover (21) showed then in addition that in disagreement with necessary requirements the exponential phase operator $\mathcal{E}=e^{-i \omega \Phi}$ (where $\Phi$ was the phase) was not unitary, as it held $\mathcal{E}^{\dagger} \mathcal{E} u_{0} \equiv 0$ for any state of zero energy if the third assumption was involved. It indicated that the given Hilbert space was not complete to represent the evolution of a corresponding physical system quite regularly.

Many attempts have been done during the 20th century to remove the last two deficiencies. The reason of having been unsuccessful may be seen in the fact that practically in all these attempts both these shortages were regarded and solved as one common problem. The corresponding solution has been obtained only recently (see Refs. $(22 ; 23)$ ) when it has been shown that it is necessary to remove two mentioned shortages one after the other.

The criticism of Pauli may be removed if the Hilbert space is extended in the accord to the time-dependent Schrödinger equation. E.g., as to the simple system of two free colliding particles it has had to be doubled in comparison to the former third assumption (see Sec. 2) as proposed by Lax and Phillips (12; 13). In this case the corresponding Hilbert space must 
consist of two mutually orthogonal subspaces $\left(\mathcal{H}=\Delta^{-} \oplus \Delta^{+}\right)$; being mutually related with the help of evolution operator and each of them being spanned on one basis of Hamiltonian eigenfunctions. The incoming or outgoing states of two particles must belong always to the different orthogonal subspaces.

As to the criticism of Susskind and Glogower the Hilbert space (extended already to solve Pauli's problem) should be further doubled and formed by combining two mutually orthogonal subspaces corresponding to systems with opposite angular momentums. These subspaces must be bound together by adding the action of exponential phase operator to link together the vacuum states (corresponding to minimum energy in individual subspaces), as it was proposed in principle already by Fain (24) also in 1967; see (22; 23). Some other points concerning the Hilbert space corresponding to the Schrödinger equation (or to the hidden-variable theory) will be mentioned in Sec. 8 .

\section{Experimental data and two quantum theories}

The Copenhagen alternative has been often denoted as supported by different experimental data. However, in all such cases only the assumptions corresponding to the Schrödinger equation (i.e., to the hidden-variable theory) have been practically tested; without the last two assumptions (forming Copenhagen alternative) having been taken into account. And therefore, none of two quantum alternatives may be excluded on the basis of the experiments practically available until now.

And it has been shown in the preceding that practically the same has held also in the case of EPR experiment. Also the original statement of Belinfante that the two given quantum alternatives has had to lead to different predictions has been shown as mistaking (see Sec. 4). Having discovered this fact many years ago it was quite natural for us to ask: When in the EPR experiment the same predictions might be obtained for the so different physical concepts, would it not be possible to find an experiment where the predictions would be different? And after a preliminary theoretical analysis the measurement of light transmission through three polarizers seemed to represent suitable way.

The corresponding experiments have been performed and the results (see Refs. $(25 ; 26)$ ) have been obtained being fundamentally different from the predictions of the Copenhagen quantum mechanics as may be seen from Figs. 1 and 2 taken from Ref. (26); see also $(9 ; 27 ; 28)$. In Fig. 1 the pairs of angles $\alpha$ and $\beta$ giving the smallest coincidence probabilities for a chosen $\alpha$ have been shown; angle $\alpha$ having been represented on horizontal axis and corresponding angle $\beta$ on vertical axis.

The values of light transmission through the polarizer triple for the given angle pairs (shown in Fig. 1) are represented by individual points on dashed line in Fig. 2. The full line represents then the prediction of the standard theory based on the Copenhagen quantum alternative. It may be seen that the existence of two maximums is predicted just where the experimental data show two deep minimums. Predictions similar to the obtained results may follow from the phenomenological polarization theory of Stokes (see (26)) but sufficient agreement with data may be hardly obtained on such a basis.

It is possible to say that the given results have opened also a way to a more realistic theory of polarization phenomena. The polarization process should be interpreted probably as a complex process; consisting practically at least of two main different successive steps: the influence of the enter layer and of the whole medium of a given polarizer. The given problem 


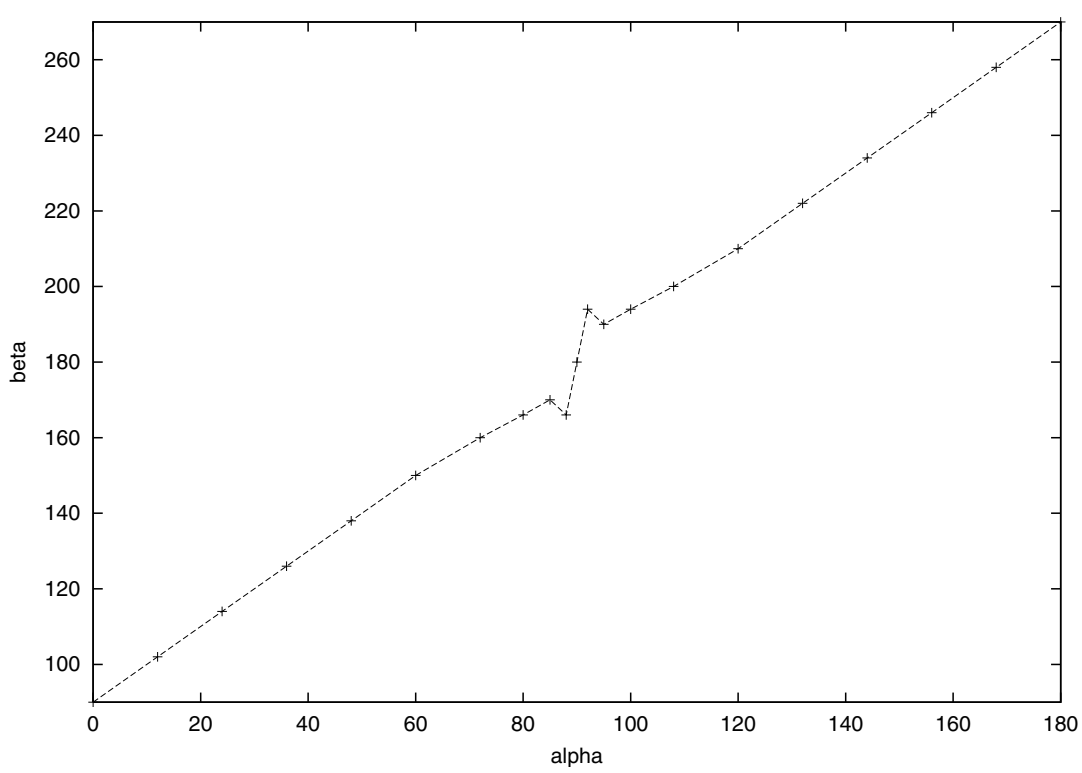

Fig. 1. The pairs of angles $\alpha$ and $\beta$ (used for the measurement shown in Fig. 2)

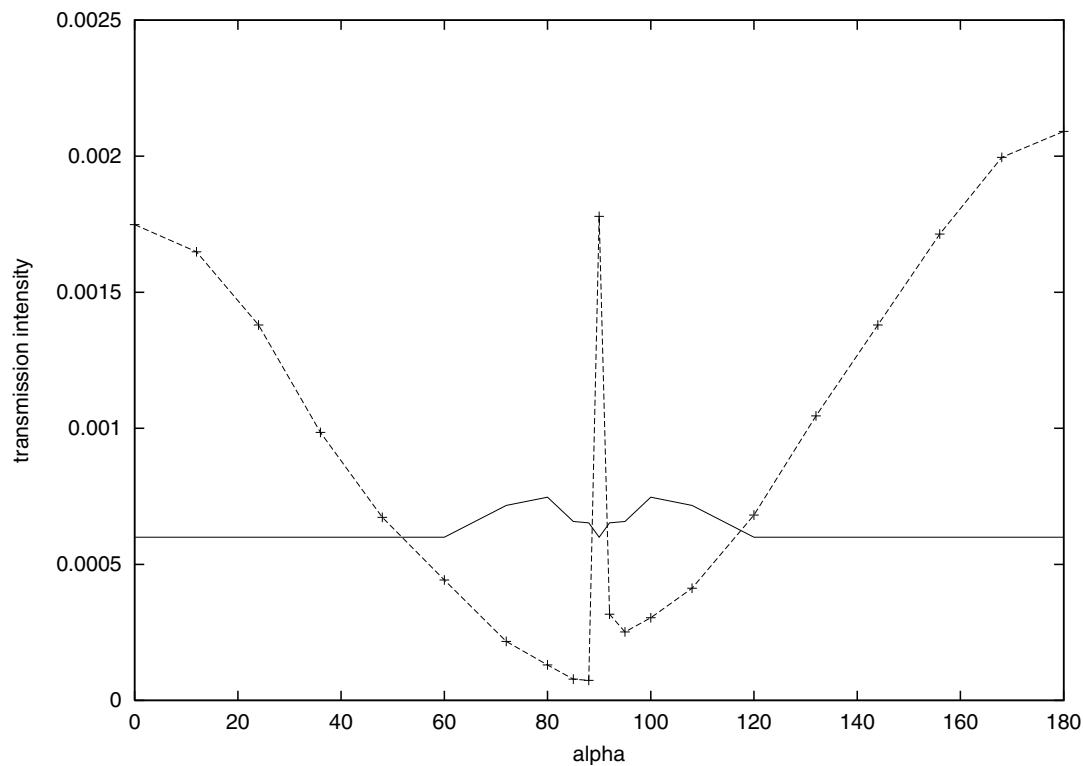

Fig. 2. Light transmission through three polarizers (for $\alpha-\beta$ pairs shown in Fig. 1); experimental data - points on dashed line; quantum-mechanical prediction - full line. 
has been studied already theoretically on such a basis by us; however, definite results have not yet been gained.

\section{Schrödinger equation and classical physics}

As already mentioned the hidden-variable theory has been practically identical with the Schrödinger equation when any other conditions deforming the physical interpretation of original solutions have not been added. And one should ask what is to be understood under the term of hidden variables. Especially it is necessary to answer the question whether some hidden variables exist in the solutions of Schrödinger equation.

The problem concerning the hidden variables in the Schrödinger equation was discussed since the beginning of the Copenhagen quantum mechanics; their existence was, however, refused by von Neumann in 1932 (29). Even if G.Hermann (30) showed already in 1935 that the proof of von Neumann was to be denoted as circle proof the corresponding discussion was renewed only after D. Bohm (5) showed that some parameter existed already in the solution of the simplest Schrödinger equation. However, the actual meaning of such parameters remained unclear.

To answer the question what is the actual essence of "hidden variables" one must ask what is the assumption basis on the grounds of which the Schrödinger equation may be derived, when at least in some cases the predictions identical with classical ones may be obtained (14). And the answer has been given by Hoyer (31) and Ioannidou (32) who have shown that it has been possible to derive it if the set of all basic solutions of Hamilton equations has been considered together with the set of all statistical superpositions of these solutions, limited only by a rather weak condition.

It is then necessary to distinguish between the basic solutions of Eq. (1) and their superpositions, when the basic solutions (or states) are given by

$$
\psi_{E}^{b}(x, t)=\psi_{E}(x) e^{-i E t}
$$

where $\psi_{E}(x)$ is eigenfunction of corresponding Hamiltonian (see Eq. (3)). The basic solutions of Schrödinger equation (characterized always by one Hamiltonian eigenfunction only) represent the so called "pure" states and correspond to individual solutions of the Hamilton equations. It does not hold in opposite direction in the case when discrete states are involved (33). In such a case only a limited subset of solutions of Hamilton equations may represent pure states in the quantum alternative.

The Schrödinger equation is linear differential equation and consequently any superposition of basic states represents its solution, too. These superpositions must be interpreted, however, always as "mixed" states; never as "pure" states. And the parameters characterizing a concrete statistical distribution of corresponding basic states have been denoted earlier as hidden parameters. They may be in principle represented by the function $\alpha(E)$ having continuous as well as discrete parts that fulfills the condition $\Sigma_{E}|\alpha(E)|^{2}=1$ if all solutions are normalized to unit value. The corresponding statistical combination (mixed state) is then represented by the solution of Schrödinger equation:

$$
\psi(x, t)=\sum_{E} \alpha(E) \psi_{E}^{b}(x, t)
$$


where each basic (pure) state conserves the corresponding energy value $E$. The average energy of the corresponding superposition equals $\sum_{E}|\alpha(E)|^{2} E$ and is also conserved. It follows on the basis of the mentioned derivation of Schrödinger equation from Hamilton equation that the function $|\alpha(E)|^{2}$ should fulfill some limiting condition (e.g., corresponding to Boltzmann statistics, see (31)) if the mentioned equivalence between Schrödinger equation and classical physics is to hold.

As to the Hilbert space the vectors of which may represent the time dependent solutions of Schrödinger equation it must contain always at least two different subspaces (each spanned on one set of Hamltonian eigenvectors) enabling to represent corresponding time flow. These subspaces must be mutually related with the help of evolution operator $U(t)=e^{-H t}$ defining the passage from one subspace to the other (see also Sec. 5). In the case of discrete Hamiltonian energy spectrum this pair of subspaces may form the infinite chain of identical pairs related by evolution operator (for more details see $(22 ; 23)$ ). If the system consists of a greater number of interacting objects each of the given subspaces may have a more complicated structure.

All basic (or pure) states are represented by mutually orthogonal vectors forming the basis of individual subspaces and corresponding to states that may be described also by Hamilton equations. Any mixed state (superposition of basic states) corresponds then to a statistical distribution of classical states, and the values $|\alpha(E)|^{2}$ represent the corresponding probabilities of individual states. It means that the hidden-variable theory (or the Schrödinger equation) can supersede also the classical physics if one admits that macroscopic physical systems exhibit discrete energy values, too; the fact being fully acceptable but experimentally unverifiable.

However, a given basic physical state is not fully determined by total energy value $E$. The other value that is conserved during the evolution is angular momentum $L$ which cannot be usually uniquely defined in an initial state. It is, of course, possible to choose the coordinate system in which the components $\left\langle L_{1}\right\rangle=\left\langle L_{2}\right\rangle=0$ and the given state may by represented by a superposition containing states with different values of $\left\langle L_{3}\right\rangle$. In a two-particle system the given value may be substituted by their minimal mutual distance $q$ during the evolution and the given superposition is characterized (in addition to $\alpha(E)$ ) also by the function $\alpha(q)$; see also the end of Sec. 8 .

\section{Schrödinger equation and corresponding Hilbert space}

All solutions of Schrödinger equation may be represented by the vectors of Hilbert space. It must have, however, the corresponding structure as mentioned in preceding. We shall demonstrate basic characteristics of this space with the help of the physical system consisting of two zero-spin particles which represents the smallest system exhibiting time evolution. Its behavior in the center-of-mass system may be described by Schrödinger equation (1) with the Hamiltonian

$$
H=\frac{p_{j}^{2}}{2 m}+V\left(q_{j}\right)
$$

where $m$ is the reduced mass of the given particle pair and $q_{j}$ and $p_{j}$ are the operators of relative coordinates and momentum components of one particle (in the center-of-mass system); they are assumed to fulfill the following relations

$$
\left[q_{j}, p_{k}\right]=i \delta_{j k}, \quad\left[p_{j}, p_{k}\right]=\left[q_{j}, q_{k}\right]=0 .
$$


Introducing two other operators

$$
Q=q_{j}^{2}, \quad R=\frac{1}{2}\left\{p_{j}, q_{j}\right\}
$$

and assuming $V\left(q_{j}\right)=V(Q)$ (i.e., the mutual potential between particles depends on their distance only) one can write further

$$
\begin{gathered}
i\left[H, q_{k}\right]=\frac{p_{k}}{m}, \quad i\left[H, p_{k}\right]=-2 q_{k} \frac{d V(Q)}{d Q}, \\
i[H, Q]=\frac{2}{m} R, \quad i[H, R]=2 H-V(Q)-Q \frac{d V(Q)}{d Q} .
\end{gathered}
$$

It is also possible to introduce angular-momentum operators fulfilling relations

$$
L_{i}=\varepsilon_{i j k}\left[q_{j}, p_{k}\right], \quad\left[L_{i}, H\right]=0 ;
$$

and further operator $L$ :

$$
L=L_{i}^{2}, \quad\left[L, L_{i}\right]=[L, H]=0 .
$$

The basic properties of a given physical system are characterized by the expectation values of the operators $\langle H\rangle$ and $\langle L\rangle$; and the basic space orientation by expectation values $\left\langle L_{j}\right\rangle$. Any time-dependent solution of the Schrödinger equation may be then represented uniquely by one trajectory in the corresponding Hilbert space. And it may be suitable to choose the special coordinate system in which $<L_{1}>=<L_{2}>=0$ (or $<q_{3}>=0$ ) and further also $\left\langle q_{1}\right\rangle=0$ and $\left\langle p_{2}\right\rangle=0$ if $\langle R\rangle=0$.

As to the structure of corresponding Hilbert space there is a significant difference between the solutions belonging to continuous or discrete spectra of Hamiltonian. We shall start with the continuous spectrum. In this case the instantaneous states with negative (positive) $\langle R\rangle$ values represent incoming (outgoing) states belonging to two different Hilbert subspaces (both being spanned on the full set of the same Hamiltonian eigenfunctions). And it is necessary to assume that these subspaces are mutually orthogonal as the given states are physically well distinguishable. It is also useful to define the time operator $T$ fulfilling the condition

$$
i[H, T]=1
$$

with the expectation value $t=0$ corresponding to the state characterized by $\langle R\rangle=0$. The whole trajectory may be obtained when the evolution operator

$$
U(t)=e^{-i H t}
$$

is applied to this initial state; the incoming (outgoing) states corresponding to negative (positive) values of $t$.

To obtain a reasonable description of an evolving physical system it is necessary always to hold $\lim _{Q \rightarrow \infty} V(Q)=0$. If the system consists of two free particles there is one-to-one correspondence between the operators $T$ and $R$ that may be specified for some concrete shapes of $V(Q)$ (34). And for a given Hamiltonian any time-dependent trajectory is uniquely defined by the function $\psi(x, 0)$; being fully determined by the values of $q_{2}(0)$ and $p_{1}(0)$ when the mentioned coordinate system has been made used of. 
The corresponding mathematical model describes suitably usual collision experiments. However, in the given experiments (for any energy value $E$ from the continuous spectrum) the collision behavior may be described only as a statistical combination of the states with different values of $q_{2}(0)$. The frequency of these individual collision events is then proportional to the values of impact parameter (distance of initial momentum direction from system mass center) that must be derived from the values obtained at a time $t_{Q}<0$ (expectation value of the operator $T$ ) for which $\langle V(Q)\rangle$ may be fully neglected against $<H>$.

If the influence of potential energy decreases monotony with $Q$ it is possible to assume for sufficiently high values of $\left|t_{Q}\right|$ that the particle moves along a straight line that may be uniquely determined for any state characterized by the values of $q_{2}(0)$ and $p_{1}(0)$. And the distance of this straight line from the parallel one going directly through the corresponding coordinate center corresponds then to the original impact parameter for the given pair of colliding particles. And it is possible to assume further that these impact parameters will be equally distributed in the plane perpendicular to the given direction and that the corresponding value of the impact parameter $b$ will represent the weight of the corresponding state in the given experiment.

In the given coordinate system the straight line corresponding to initial momentum transects the $q_{2}$-coordinate at the value

$$
y=\frac{q_{2}\left(t_{Q}\right) p_{1}\left(t_{Q}\right)-q_{1}\left(t_{Q}\right) p_{2}\left(t_{Q}\right)}{p_{1}\left(t_{Q}\right)}
$$

and it holds also

$$
\frac{b}{y}=\frac{p_{1}\left(t_{Q}\right)}{p\left(t_{Q}\right)}
$$

The numerator in Eq. (15) represents one component of angular momentum. And if one takes into account that the angular momentum component commutes with Hamiltonian (and is conserved during the evolution) it holds further

$$
b=\frac{\left|L_{3}\right|}{p\left(t_{Q}\right)}
$$

or

$$
b=\left|q_{2}(0)\right| \frac{p(0)}{p\left(t_{Q}\right)}
$$

where $p\left(t_{Q}\right)$ may be derived immediately from the corresponding energy $E$ determined with the help of values $p(0)=\left|p_{1}(0)\right|$ and $q_{2}(0)$. The frequency of a state characterized by the values of $q_{2}(0)$ and $p_{1}(0)$ in the given experiment is then proportional to the value of $b$. It means that the states corresponding to very small values of $\left|q_{2}(0)\right|$ become more frequent for an attractive potential and less frequent for a repulsive one.

The given weight of states characterized by the value $q_{2}(0)$ in the statistical distribution is important in the case when the interaction between colliding particles is mediated by forces at distance (e.g., Coulomb forces) as the density of states at small values of $|t|$ may be significantly influenced by the corresponding shape of potential dependence at small mutual distances. In the case of contact forces between colliding particles (which may concern strong 
interactions) one may regard corresponding tracks as linear in the whole length (before the proper collision process).

The preceding considerations have concerned the case when the Hamiltonian spectrum has been continuous. The situation is more complex in the case of discrete Hamiltonian spectrum as the zero value of $\langle R>$ repeats during periodical time evolution; four zero values existing already in any period. Each period may be then represented by two pairs of subspaces corresponding to positive and negative values of $\langle R\rangle$ which continuously repeat and may form an infinite series where individual states are characterized by different expectation values of operator $T$. More suitable operators than $R$ (represented now by goniometric type functions) may be introduced in the case of special shapes of $V(Q)$; see (34).

\section{Physical science and ontological thinking}

The human thinking in the preceding century was undoubtedly rather strongly influenced also by the progress of physical research, which was often being connected with the phenomenistic ideas of the Copenhagen quantum mechanics. The corresponding ideas differed fundamentally from the earlier ontological concept proposed by Aristotle and developed further by Thomas Aqu. in the Middle Ages, from which G. Galileo and I. Newton started when the basis of classical physics was built up and the theoretical background was formulated further by W.R. Hamilton (1805-65); and the whole modern civilization has been based in principle fully on it.

However, this train of thought started to change from the beginning of the modern age. The initial impulse was given probably by Occam's razor when the simplicity started to be decisively preferred in explaining the reality that might be often very complex. The decisive step was done, however, by R. Descartes who refused practically any ontology by his "Cogito, ergo sum"; the human thinking started to decide practically about the real existence on the basis of different observed phenomena without any deeper ontological analysis. The given phenomenism started to influence strongly also the physical science when L. Boltzmann declared the rise of uniform distribution in the systems consisting of many particles (i.e., the phenomenistic feature) as basic natural law in 1867. A further important step occurred when N. Bohr based the atom theory on two phenomenological postulates in 1913, but mainly when he proposed the Copenhagen quantum mechanics in 1927. And since Bohr's victory in the controversy with Einstein in 1935 the corresponding phenomenistic approach asserted oneself in the western world practically in all regions of human thinking and knowledge.

As to the microscopic physics region the given research has been divided practically into two different branches. One part of physicists has continued in the research on the earlier ontological basis without taking the conclusions of Copenhagen alternative practically into account when they looked for the solutions of Schrödinger equation based on the same Hamiltonian as the classical physics; any additional deforming assumptions having not been added.

The other (lesser) part of these physicists has looked then mainly for arguments to persuade the human community about the validity of quantum paradoxes. This part of physicists was making use of the successes of modern research based on the solutions of Schrödinger equation often as the decisive support of their conviction, without respecting the fact that any of the assumptions added by Bohr (see Sec. 2) has not been taken into account when only solutions of Schrödinger equation have been looked for and directly interpreted. In 
such a case the existence of the correspondingly extended Hilbert space (as described in Sec. 8) has been automatically assumed in the interpretation of physical processes; no deformation according to Bohr's assumptions has been applied to the corresponding solutions of Schrödinger equation.

It follows from the presented results that the phenomenistic approach has brought the human knowledge to misleading conclusions. It should be abandoned and the common human thinking should return again to the earlier ontological approach in studying the matter world. It might open the new way to deeper understanding not only of the fundamental structure of matter, but also of other problems of human kind.

\section{Conclusion}

Let us introduce yet the short summary of all previous results:

The Copenhagen quantum mechanics may be denoted as falsified on the basis of logical contradictions as well as of experimental data (light transmission through a polarizer triple). Schrödinger equation itself (or hidden-variable theory) should be preferred not only to the Copenhagen quantum mechanics but also to the classical physics; it may be applied to the whole matter reality (microscopic as well as macroscopic).

The basic solutions of Schrǒdinger equation (characterized by one Hamiltonian eigenfunction only) fulfill always classical properties. It does not hold in opposite direction in the case of discrete Hamiltonian spectrum; some solutions of Hamilton equations having not any quantum counterpart.

There are not in principle any hidden variables in the Schrödinger equation. In the case of superposition solutions only the statistical distribution of potential energy corresponding to individual basic states must be defined with the help of the distribution of some physical quantities in a suitable time instant.

Superposition solutions do not represent any of the so called pure states. They must be always interpreted as mixed states. There is not any gap between microscopic and macroscopic world. Any superposition represents always the set of states that may be interpreted classically (or ontologically).

And finally, one must stress that in the given controversy Einstein was right. The purely phenomenological accession to the physical reality should be refused and the ontological approach to physical problems again strongly preferred. The physics (and all science) is to become again serious problem of human knowledge and not a mere game deciding between different phenomenologically based attitudes (comp., e.g., (4)).

\section{References}

[1] N.Bohr: The quantum postulate and the development of atomic theory; Nature 121, 580-90 (1928).

[2] A.Einstein, B.Podolsky, N.Rosen: Can quantum-mechanical description of physical reality be considered complete?; Phys. Rev. 47, 777-80 (1935).

[3] N.Bohr: Can quantum-mechanical description of physical reality be considered complete?; Phys. Rev. 48, 696-702 (1935).

[4] R.G.Newton: How physics confronts reality: Einstein was correct but Bohr won the game; World Scientific (2009). 
[5] D.Bohm: A suggested interpretation of the quantum theory in terms of "hidden variables"; Phys. Rev. 85, 180-93 (1952).

[6] J.S.Bell: On the Einstein Podolsky Rosen paradox; Physics 1, 195-200 (1964).

[7] A.Aspect, P.Grangier, G.Roger: Experimental realization of Einstein-Podolsky-Rosen-Bohm Gedankenexperiment: A new violation of Bell's inequalities; Phys. Rev. Lett. 49, 91-4 (1982).

[8] M.V.Lokajíček: Einstein-Bohr controversy and theory of hidden variables; NeuroQuantology (section: Basics of Quantum Physics) 8 (2010), issue 4, 638-45; see also /arXiv:1004.3005[quant-ph].

[9] M.V.Lokajíček: Quantum theory without logical paradoxes; Concepts of Physics 6, No.4, 581-604 (2009); see also /arxiv:09050140[quant-ph].

[10] M.V.Lokajíček: Physical theory of the twentieth century and contemporary philosophy; Concepts of Physics 4 (2007), no.2, 317-39; arXiv:quant-ph/0611069.

[11] F.J.Belinfante: A survey of hidden-variable theories; Pergamon, Oxford, p. 283 (1973).

[12] P.D.Lax, R.S.Phillips: Scattering theory; Academic Press (1967).

[13] P.D.Lax, R.S.Phillips: Scattering theory for automorphic functions; Princeton (1976).

[14] E.Schrödinger: Quantisierung als Eigenwertproblem; Ann. Phys. 79, 361-76; 489-527; 80, 437-90; 81, 109-39 (1926).

[15] J.F.Clauser, A.Shimony: Bell's theorem: experimental tests and implications; Rep. Prog. Phys. 41, 1881-91 (1978).

[16] M.V.Lokajíček: Locality problem, Bell's inequalities and EPR experiments; /arXiv:quant-ph/9808005 (1998).

[17] M.Hillery, B.Yurke: Bell's theorem and beyond; Quantum Semiclass. Optics 7, 215-227 (1995).

[18] M.Revzen, M.Lokajíček, A. Mann: Bell's inequality and operators' noncommutavity; Quantum Semiclass. Optics 9, 501-6 (1996).

[19] M.V.Lokajíček: Semiclassical interpretation of microscopic processes; arXiv:quant-ph/0511184.

[20] W.Pauli: Die allgemeinen Prinzipien der Wellenmechanik; Handbuch der Physik XXIV, Springer, Berlin, p. 140 (1933).

[21] L.Susskind, J.Glogover: Quantum mechanical phase and time operator; Physics (Long Island City, N.Y.) 1, 49-61 (1964).

[22] P.Kundrát, M.Lokajíček: Three-dimensional harmonic oscillator and time evolution in quantum mechanics; Phys. Rev. A 67, art. 012104 (2003).

[23] P.Kundrát, M.Lokajíček: Irreversible time flow and Hilbert space structure; New Research in Quantum Physics (eds. Vl.Krasnoholovets, F.Columbus), Nova Science Publishers, Inc., pp. 17-41 (2004).

[24] V.Fajn: Quantum harmonic oscillator in phase representation and the uncertainty relation between the number of quanta and the phase (in Russian); J. Exp. Theor. Phys. 52, 1544-8 (1967).

[25] J.Krása, J.Jiřička, M.Lokajíček: Depolarization of light by an imperfect polarizer; Phys. Rev. E 48, 3184-6 (1993).

[26] J.Krása, M.Lokajíček, J.Jiřička: Transmittance of laser beam through a pair of crossed polarizers; Phys. Letters A 186, 279-82 (1994).

[27] M.V.Lokajíček: Hidden-variable theory versus Copenhagen quantum mechanics; Frontiers of Fundamental and Computational Physics - Proc. of the Ninth International 
Symposium, January 2008, Udine and Trieste, edited by Sidharth B.G. et al., Conference Proceedings, No. 1018, American Institute of Physics, pp. 40-5 (2008); see also extended version: /arXiv:0711.3977v3[quant-ph].

[28] M.V.Lokajíček: Hidden-variable theory contra Copenhagen quantum mechanics; Proc. of 16th Conf. of Czech and Slovak Physicists (ed. J.Kř́žz), MAFY Hradec Kralove, pp.406-10 (2009).

[29] J.von Neumann: Mathematische Grundlagen der Quantenmechanik; Springer (1932).

[30] Grete Herrmann: Die Naturphilosophischen Grundlagen der Quantenmechanik; Abhandlungen der Fries'schen Schule 6 (1935), 75-152.

[31] U.Hoyer: Synthetische Quantentheorie; Georg Olms Verlag, Hildesheim (2002).

[32] H.Ioannidou: A new derivation of Schrödinger equation; Lett. al Nuovo Cim. 34, 453-8 (1982).

[33] M.V.Lokajíček: Schrödinger equation, classical physics and Copenhagen quantum mechanics; New Advances in Physics 1, No. 1, 69-77 (2007); see also /arxiv/quant-ph/0611176.

[34] M.V.Lokajíček: Realistic theory of microscopic phenomena; a new solution of hidden-variable problem; /arxiv:quant-ph/9811030. 


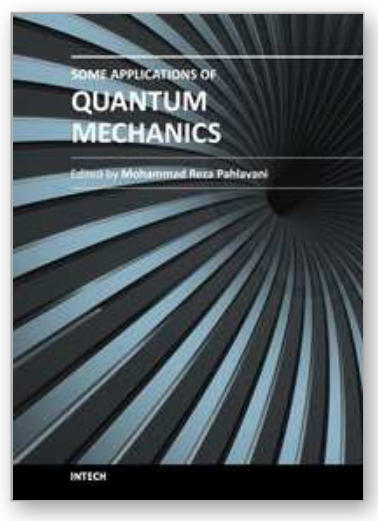

\author{
Some Applications of Quantum Mechanics \\ Edited by Prof. Mohammad Reza Pahlavani
}

ISBN 978-953-51-0059-1

Hard cover, 424 pages

Publisher InTech

Published online 22, February, 2012

Published in print edition February, 2012

Quantum mechanics, shortly after invention, obtained applications in different area of human knowledge. Perhaps, the most attractive feature of quantum mechanics is its applications in such diverse area as, astrophysics, nuclear physics, atomic and molecular spectroscopy, solid state physics and nanotechnology, crystallography, chemistry, biotechnology, information theory, electronic engineering... This book is the result of an international attempt written by invited authors from over the world to response daily growing needs in this area. We do not believe that this book can cover all area of application of quantum mechanics but wish to be a good reference for graduate students and researchers.

\title{
How to reference
}

In order to correctly reference this scholarly work, feel free to copy and paste the following:

Miloš V. Lokajiček (2012). Einstein-Bohr Controversy After 75 Years, Its Actual Solution and Consequences, Some Applications of Quantum Mechanics, Prof. Mohammad Reza Pahlavani (Ed.), ISBN: 978-953-51-0059-1, InTech, Available from: http://www.intechopen.com/books/some-applications-of-quantum-mechanics/einsteinbohr-controversy-after-75-years-its-actual-solution-and-consequences

\section{INTECH}

open science | open minds

\section{InTech Europe}

University Campus STeP Ri Slavka Krautzeka 83/A 51000 Rijeka, Croatia Phone: +385 (51) 770447

Fax: +385 (51) 686166 www.intechopen.com

\section{InTech China}

Unit 405, Office Block, Hotel Equatorial Shanghai No.65, Yan An Road (West), Shanghai, 200040, China 中国上海市延安西路65号上海国际贵都大饭店办公楼405单元 Phone: +86-21-62489820

Fax: $+86-21-62489821$ 
(C) 2012 The Author(s). Licensee IntechOpen. This is an open access article distributed under the terms of the Creative Commons Attribution 3.0 License, which permits unrestricted use, distribution, and reproduction in any medium, provided the original work is properly cited. 